\title{
Advances in next era cloud-empowered computing and techniques
}

\author{
Jason C. Hung ${ }^{1}$ • Gangman $\mathbf{Y i}^{2}$
}

Published online: 5 July 2017

(C) Springer Science+Business Media, LLC 2017

\section{Introduction}

Cloud computing is defined as a pool of virtualized computing resources. Based on the virtualization, the cloud computing paradigm allows workloads to be deployed across physical machines via virtual resources. A cloud computing platform supports redundant, self-recovering, and scalable programming models that allow workloads to recover from many inevitable hardware/software failures. In a mechatronic and embedded cloud computing platform, software is migrating from an embedded system into a network, providing users with an anytime, anywhere access to programs and data on the mechatronic or embedded systems. This special issue focuses on the use of cloud-based technologies to meet new compute-intensive and data-intensive scientific challenges that are not well served by the current supercomputers, grids, and highperformance computing clusters.

This special issue includes high-quality technical papers addressing research achievements, practices, and challenges for shaping the cloud-empowered computing and techniques in the next generation. Original and research articles are consisting of all aspects of including theoretical studies, practical applications, new social technology, and experimental prototypes.

\footnotetext{
$凶$ Gangman Yi

gangman@dongguk.edu

Jason C. Hung

jasonhung2013@gmail.com

1 Department of Information Technology, Overseas Chinese University, Taichung 407, Taiwan

2 Department of Multimedia Engineering, Dongguk University, Seoul 04620, South Korea
} 


\section{Related works}

Yeji Kim et al. present a hand gesture interaction framework for virtual experimental applications that allow multiple users to interact with NUI (Natural User Interface). For this purpose, the framework operates in the cloud environment enabling applications to run in a $3 \mathrm{D}$ virtual environment with the client-server structure for multiple client access and communication [1,2].

Yan Li et al. describe a short path planning algorithm for complex indoor spaces using multiaccess point topology analysis. The demand for effective navigation in complex indoor environments is becoming more apparent because even a person who is familiar with the specific place or not when a someone needs effective guidance in the complicated indoor in an emergency situation $[3,4]$. The algorithm acquires navigation path by combining spatial analysis and interiors of especially indoor environments. The experimental comparisons show that the algorithm gains reasonable performance in both running time cost and path distance.

Daesung Moon et al. propose a decision tree-based IDS against APT attacks. The Advanced Persistent Threats (APT) attack has great concerns about network security and cyber-defense techniques, because it can invade general security countermeasures and analyzed vulnerabilities of the target systems continuously [5,6]. This paper proposed a decision tree-based IDS applying behavior analysis for detecting APT attacks. The proposed system analyzes the processes of systems and abnormal behaviors of the current networks, which are learned through the decision tree, and it can detect intrusion based on behavioral information.

Fairouz Fakhfakh et al. address the problem of scheduling of workflows in the IaaS clouds. The objective is the minimization of cost under a deadline constraint. The specific problem the authors address is the possible dynamic change in the workflow at runtime. This paper introduces a provisioning algorithm based on the meta-heuristic optimization technique Particle Swarm Optimization (PSO), and it shows better results in solving task assignment than other algorithms [7]. Moreover, the authors give a motivating example from a business workflow domain, where the subset of tasks can be inserted, deleted, or replaced due to exceptions during workflow execution caused by the changing external conditions or business requirements.

Yong-hwan Kim et al. describe a new distributed LTE/EPC network architecture based on SDN, NFV, and cloud computing, with distributed P-GWs and centralized control plane in LTE/EPC networks. The authors designed the proposed scheme with the three factors for the re-designing of LTE/EPC network which includes distributing P-GWs closer to the UEs, virtually centralizing control plane, and separation of the control and data planes. The proposed idea is very reasonable and helpful for alleviating the mobile traffic loads, and the description of the paper is also well given. In particular, it is expected that the proposed route optimization strategy for internal traffic exchanged between LTE users will be very useful in real networks $[8,9]$. The proposed scheme is evaluated and justified by comparison with the conventional LTE/EPC network scheme regarding data processing volume, handover latency, and the number of valid data sessions.

Youngho Jo et al. propose new SIIS (Social Identification-Issuing System) which samples micro-movement using co-movement issued social IDs and social behaviors. 
The goal of our study is to recognize the social behavior by using co-movement with other people by micro-movements and determining the social identification of group showing the same behavior. In order to do such process, the participant' $\mathrm{s}$ behavior was analyzed while determining the interaction differences between the interaction mode of face-to-face condition and personal mode of the mobile phone interaction. This research could be used in determining social groups and provide the information to support a service to social media—such as Facebook, Twitter, Instagram, or other social media services_or lifelogging, such as Sony Lifelog, Fitbit Charge HR, and other similar services. Data for distinguishing co-movement can expand the entire lifelogging data such as heartbeat, behavior, and consumption contents. By forming social groups with many people's data, this system can be expanded to deduce patterns and trends of public $[10,11]$.

Longbin Chen et al. present a hybrid memory system for embedded devices based on BSNs in telehealth. The energy-saving issues for the embedded devices based on BSNs are a critical matter for wearable devices and wireless sensor networks, especially telehealth $[12,13]$. This paper designed an ecosystem for telehealth, such as memory storage of embedded devices and data storage of telehealth data centers. It designed several resource allocation algorithms based on PCM with flash memory using dynamic programming and heuristics to enable the proposed ecosystem harmoniously.

Min-Kuan Chang et al. describe the problem of the transmission behavior of nodes in an IoT environment. The authors proposed to apply the theory of continuum percolation to analyze the connectivity of nodes in such an environment. Also, the authors provided a solution to enhance the performance of connectivity between nodes in an IoT. This paper firstly investigates the possibilities between two nodes communication and bonds percolation in a two-dimensional lattice.

Shih-Jung Wu et al. propose an opinion-mining system for Chinese Internet online forums [14]. It can effectively analyze a large number of articles and provides the correct evaluation information and supportive decision making to the users [15]. This system not only can rapidly acquire the objective assessment information but also can track whether it is the long-term behavior of malicious criticism by the reviewer and identify the abnormal evaluation. The authors suggest word-hyphenation algorithms for a semiautomatic Chinese opinion-mining system. Firstly, extracting new Internet words may become new opinion elements from an article posted on an Internet forum. And secondly, it addresses the possible change in the polarity or meaning of an opinion word when that opinion word is combined with a specific word or concatenated with other opinion words.

Yan Pei addresses a machine learning algorithms based on orthogonal transformation by using IEC (interactive evolutionary computation) to solve the selection problem. Interactive evolutionary computation (IEC) is a general term for methods of evolutionary computation that use human evaluation which enables optimization approach that embeds human subjective evaluation into a process of system optimization [16]. An image compression problem using principal component analysis is introduced to study the proposed method as interactive DE which is differential evolution is one of population-based optimization algorithms [17]. 
Chee Sun Won and Seung-Won Jung describe an image resizing proposal for supporting different spatial resolutions. They formulate image resizing as a MAP (maximum a posteriori) optimization problem with a Gibbs energy function to reduce computational complexity using a sub-optimal solution of the MAP criterion with a deterministic implementation of the Metropolis algorithm [18].

Byoung Cheul Kim et al. address a new 3D gaze-tracking method using Kinect v2, which combines 3D eye and facial gaze vectors. Because of the reliability of Kinect v2 accuracy, the method is very suitable with accurate 3D NUI, and also, it can use intelligent vehicle apps. There is several 3D-based gaze-tracking methods despite the computation overhead, because of better accuracy results compared to 2D based $[19,20]$. The result of an experiment in this paper shows the outstanding accuracy based on the proposed combined gaze-tracking system. The average RMS error was approximately 23 pixels for a Full HD resolution.

Zhen Li et al. present a mechanism discovering evasive attacks on HTTP. The authors use some techniques to improve the performance. They focused on how network attacks based on exploit HTTP hides their malicious activities, and they suggested called, Evasive Network Attack Forensic System (ENAFS), which can effectively discover evasive network attacks on HTTP. Moreover, it integrally draws the attack samples and their metadata for further analysis [21]. Also, the experimental results show that the proposed mechanism copes well with HTTP evasive behavior.

Jeong Kyu Lee et al. propose a cloud analysis-based enhanced ransomware, called CloudRPS. As we know, it is difficult to detect and judge a malicious behavior quickly because it continues to mutate and evolve [22,23]. The CloudRPS is installed in a cloud system that can analyze the network, file system, the server logs to defend against the malicious attacks. The database collects data from an external cloud system and then transmits it to the server monitor. The server monitor detects a malicious ransomware and an abnormal behavior through monitoring. The behavior detection through server monitor tested through the ransomware inspector, and the suspicious files delivered to the malware analyzer. The malware analyzer verifies whether it is or not. The ransomware that ruled as malicious sends it to the classifier. It categorizes the ransomware by threat level, and this information will send to the database.

Wei-Chen Wu presents a secure method for message passing among vehicles in VANET (Vehicular Ad hoc Network) clouds. This method includes these phases: Registration, Multicast Authentication, Session Key Generation, RSU-Based Key Exchange, Key Request from Other RSUs, Anonymous Request, and Secret Push Messaging Transmission. The experiment shows that our scheme reduces more than $25 \%$ of the message delivery times at least, and when the vehicle passes through more roadside units, more than half of the message can reduce delivery times if the number of RSUs is more than 50 .

Hae Min Moon et al. describe an approach for lower complexity interpolation that is based on linear and cubic convolution method in image resolution up conversion [24]. They propose an interpolation method suitable for the space-based resolution module that creates high-quality images with relatively few computations and hardware resources without a coefficient generator unit. They propose an interpolation method suitable for space-based resolution modules that produce high-quality images with relatively little computation and hardware resources without a coefficient generator 
unit. Thus, it is more suitable than the bicubic convolution interpolation method for hardware implementation.

Yan Zhuang et al. propose a novel data analysis approach to predict next checkin locations on Next Foursquare. Foursquare is a local search-and-discovery service mobile app which provides search results for its users, and it provides personalized recommendations of places to go to near a user's current location based on users' "previous browsing history, purchases, or check-in history" [25]. They suggest that a relatively simple technique is proposed and studied based on Foursquare [26], which can guess the most likely subsequent check-in location by computing the probabilities of check-in occurrences.

Yan Zhuang et al. present a novel methodology for predicting the next turn at road junction based on big traffic data. The next- turn prediction model predicts the turn which is most likely to proceed from the road connection point after traveling a specific pattern on the road [27]. They present a simple probabilistic model that predicts the next position of a road user based on the "segment concept" extracted from the historical trail accumulated by the user over time in several data archives. Their experimental results show that there is some influence on the length of the past trajectory the user has moved to the accuracy of the next-turn prediction.

Shih-Hao Chang and Mao-Sheng Hung propose a software-defined wireless network architecture enable high performance in the next- generation ship area network (ShAN). They claimed that both network (Layer 2) and routing (Layer 3) protocols would be required to guarantee data transmission. They proposed a new network architecture, which combines Self-Organizing Time Division Multiple Access (SOTDMA) [28] and Software-Defined Wireless Networking (SDWN) [29] advantages to reduce end-to-end transmission delay to achieve high performance in ship area networks. The full design of high-performance SDWN using the proposed architecture will be an effective tool for future ship area networks which supports SOTDMA module based on OPNet.

Qingsong Feng et al. describe a proposal for anomaly detection of spectrum in wireless communication via deep-structure auto-encoder which is neural networks to detect the anomalies of the spectrum. It relies on the signal spectral features and auto-encoders with a deep structure as a one-class classifier [30]. The time-frequency diagram of suggested idea is acted as the feature of the learning model. The comparison of the representation learned by the proposed model between the reconstructed data restored from these representations suggests that their model based on the Deep AutoEncoder can extract useful features from raw data without artificial selection.

Eunmi Choi and Wonhee Cho propose preprocessing methods for big data in vehicle driving data applying MapReduce. Although big data preprocessing is essential, there has been a lack of research works with case studies [31]. The proportion of DTG sensing data points including any errors was up to $27.09 \%$. Compared to the traditional brute-force way to detect, proposed method performs $71.1 \%$ additional detection effect.

Yi-Hsuan Lee et al. present the proposal for efficiently resources allocation applying Distributed Resource Allocation (DRA) to host distributed tasks for reliability, availability, and cost $[32,33]$. This research also adopts a task grasping method to minimize the communication waiting for time effort between different clouds in the Federation 
cloud. The experimental results demonstrate that the proposed DRA approach can improve resource utilization and has superiority in the profit in the federated cloud.

Jieh-Ren Chang et al. describe the fuzzy preprocessing classifiers to extract the local information from the corrupted image. The classifier categorizes broken pixels into five distributed impulse noises and uses a detection procedure to extract more information of the local image of the fuzzy classifier [34]. The fuzzy rule-based preprocessing classification method can identify noise from next-generation cloud computing services [35]. The performance of the proposed method verified by objective numerical measurement and subjective visual inspection. The results show that the proposed method works remarkably regarding noise suppression and detailed preservation.

Vishal Sharma et al. propose fuzzy-bee colony optimization based in ad hoc networks. The authors describe a fuzzy-based bee colony optimization algorithm is proposed which operates over cooperative trust value, cognitive relaying value and situational awareness to provide efficient cognitive relaying between the two ad hoc units, and uses a cloud server to provide coordination between the nodes. The effectiveness of the proposed scheme and its applicability is tested using simulations.

Binbin Yong et al. present an intelligent monitor system based on a cloud system with convolutional neural networks. The authors describe cloud-based system to share computation resources to reduce redundant computation based on hybrid CNNs (convolutional neural networks) $[36,37]$. The proposed system combines several trained CNNs through a cloud server, which are used to make different feature recognition based on frames from webcam stream.

Jin Wang et al. describe cluster-based dynamic routes adjustment approach for wireless sensor networks with mobile sinks. The authors presented an Energy Efficient Cluster-based Dynamic Routes Adjustment approach (EECDRA), which is concentrated to minimize the reconstruction cost for routes within the sensor nodes while maintaining optimal routes to the latest location of the mobile sinks [38]. The lifetime of the wireless sensor network can extend by the optimal route and a limited flooding of update messages to a limited number of cluster heads. The suggested cluster head rotation mechanism efficiently alleviated the hot spot problem.

Deok Gyu Lee proposes a method to divide the dangerous situation into three or more levels and recognize dangerous situations with behavior networks based on Bayesian probability. The author uses the test data measured by the sensors of a smartphone are utilized to recognize dangerous situations, and the proposed method learns according to the result of recognition [39]. This method automatically perceives the user's dangerous situation in the event of an emergency.

Jong Hyuk Park et al. present a problem to catch abnormal private data access signal for SNS security. There are various researches on SNS security related to enhancing privacy issues, including the privacy leakage and personal data sharing hazards [40] and updated analysis of security and privacy within SNS services [41]. The proposed method detects abnormal private data access signals for SNS security by analyzing consecutive private data access signals. The solution for the problem is to analyze consecutive private data access signal and to find abnormal patterns by comparing with real-time patterns using Bayesian probability. The suggested SNS security framework to detect abnormal PDA signals by predefining formal PDA signals. Formal PDA 
signals are defined by analyzing the PDA signals originating from an SNS and by grouping consecutive signals as PDA patterns.

\section{References}

1. Ahn J, Eom J-H, Nam S, Zong N, Im D-H, Kim H-G (2017) xStore: Federated temporal query processing for large scale RDF triples on a cloud environment. Neurocomputing 256(20):5-12

2. Campos R, Dias G, Jorge AM, Jatowt A (2014) Survey of temporal information retrieval and related applications. ACM Comput Surv 47(2):15

3. Guha R K, Chen W (2009) A distributed traffic navigation system using vehicular communication. In: Proceedings of 1st IEEE Vehicular Network Conference. pp 1-8. doi:10.1109/VNC.2009.5416381

4. Abbas F, Oh H (2014) A step towards user privacy while using location-based services. JIPS 10(4):618627. doi:10.3745/JIPS.01.0003

5. Virvilis N, Gritzalis D, Apostolopoulos T (2013) Trusted computing vs. advanced persistent threats: can a defender win this game? In: Ubiquitous Intelligence and Computing 2013 IEEE 10th International Conference on and 10th International Conference on Autonomic and Trusted Computing (UIC/ATC). IEEE, pp 396-403

6. Virvilis $\mathrm{N}$ et. al (2013) The big four-what we did wrong in advanced persistent threat detection?. In: IEEE 2013 Eighth International Conference on Availability, Reliability and Security (ARES)

7. Salman A, Ahmad I, Al-Madani S (2002) Particle swarm optimization for task assignment problem. Microprocess Microsyst 26(8):363-371

8. Y-h Kim, Han Y-H, Kim M, Park YS, Moon SJ, Lee JH, Choi DK (2013) Distributed mobility management scheme in LTE/SAE networks. J Korea Inf Commun Soc 38B(11):879-894

9. Kim Y-h, Han Y-H, Kim M, Park YS, Moon SJ, Lee JH, Choi DK (2014) Distributed PDN gateway support for scalable LTE/EPC networks. In: IEEE CCNC International workshop on mobility management in the networks of the future world (MobiWorld 2014)

10. Jang Y, Shin S, Lee J W, Kim S (2007) A preliminary study for portable walking distance measurement system using ultrasonic sensors. In: Proceedings of the 29th Annual International Conference of the IEEE EMBS Cité Internationale, Lyon, France, August 23-26

11. Shrivastava AK, Verma A, Singh SP (2010) Distance measurement of an object or obstacle by ultrasound sensors using P89C51RD2. Int J Comput Theory Eng 2(1):64-68

12. Hu J et al (2012) Optimizing data allocation and memory configuration for non-volatile memory based hybrid SPM on embedded CMPs. In: IPDPSW. Shanghai, China, pp 982-989

13. Meza J et al (2012) Enabling efficient and scalable hybrid memories using fine granularity DRAM cache management. IEEE Comput Archit Lett 11(2):61-64

14. Liu B (2012) Sentiment analysis and opinion mining, synthesis lectures on human anguage technologies. vol. 5, pp 1-167, 2012/05/23

15. Chen M, Yao T (2010) Combining dependency parsing with shallow semantic analysis for Chinese opinion-element relation identification. In: Universal Communication Symposium (IUCS), 2010 4th International (pp 299-305)

16. Takagi $\mathrm{H}$ (2001) Interactive evolutionary computation: fusion of the capabilities of EC optimization and human evaluation. Proc IEEE 89(9):1275-1296

17. Price K, Storn RM, Lampinen JA (2006) Differential evolution: a practical approach to global optimization. Springer, Berlin

18. Won CS, Shirani S (2011) Size-controllable region-of-interest in scalable image 360 representation. IEEE Trans Image Process 20(5):1273-1280

19. Bostanci E, Kanwal N, Clark AF (2015) Augmented reality applications for cultural heritage using Kinect. Hum Centric Comput Inf Sci 5(1):1-18

20. Lee JW, Cho CW, Shin KY, Lee EC, Park KR (2012) 3D gaze tracking method using Purkinje images on eye optical model and pupil. Opt Lasers Eng 50(5):736-751

21. Khamphakdee N, Benjamas N, Saiyod S (2014) Improving intrusion detection system based on snort rules for network probe attack detection. In: 2nd International Conference on Information and Communication Technology (ICoICT), pp 69-74

22. Jingle DJ, Rajsingh EB (2014) ColShield: an effective and collaborative protection shield for the detection and prevention of collaborative flooding of DDoS attacks in wireless mesh networks. Hum Centric Comput Inf Sci, 4(1), article. 8 
23. Narudin FA, Feizollah A, Anuar NB, Gani A (2014) Evaluation of machine learning classifiers for mobile malware detection. Soft Comput Methodol Appl 20(1):343-357

24. Lin CC, Sheu MH, Chiang HK, Liaw CS, Wu ZC, Tsai WK (2010) An efficient architecture of extended linear interpolation for image processing. J Inf Sci Eng 26:631-648

25. The introduction of Foursquare. https://en.wikipedia.org/wiki/Foursquare

26. Cramer H, Rost M, Holmquist LE (2011) Performing a check-in: emerging practices, norms and 'conflicts' in location-sharing Using foursquare. In: Proceedings of the 13th International Conference on Human Computer Interaction with Mobile Devices and Services, ACM New York, NY, USA, pp 57-66

27. Jeung H, Yiu ML, Zhou X (2010) Path prediction and predictive range querying in road network databases. VLDB J 19(4):585-602

28. ETSI (2012) Intelligent Transport Systems (ITS); STDMA recommended Parameters and Settings for Cooperative ITS; Access Layer Part. Technical report, European Telecommunications Standards Institute

29. Jarschel M, Oechsner S, Schlosser D, Pries R, Goll S, Tran-Gia P (2011) Modeling and performance evaluation of an openflow architecture. In: Proceedings of the 23rd international teletraffic congress (ITC)

30. Liu S, Greenstein LJ, Trappe W, Chen Y (2012) Detecting anomalous spectrum usage in dynamic spectrum access networks. Ad Hoc Netw 10(5):831-844

31. Lai WK, Chen YU, Wu TY, Obaidat MS (2014) Towards a framework for large-scale multimedia data storage and processing on Hadoop platform. J Supercomput 68(1):488-507

32. Malik S, Huet F, Caromel D (2012) Latency based dynamic grouping aware cloud scheduling. In: 26th International Conference on Advanced Information Networking and Applications Workshops, pp 1190-1195

33. Cenk Erdil D (2013) Autonomic cloud resource sharing for intercloud federations. Futur Gener Comput Syst 29(7):1700-1708

34. Lien CY, Huang CC, Chen PY, Lin YF (2013) An efficient denoising architecture for removal of impulse noise in images. IEEE Trans Comput 62(4):631-643

35. Duan F, Zhang YJ (2010) A highly effective impulse noise detection algorithm for switching median filters. IEEE Signal Proc Lett 17(7):647-650

36. Chen L, Wang S, Fan W, Sun J, Naoi S (2015) Beyond human recognition: a CNN-based framework for handwritten character recognition. In: Proceeding of 2015 3rd IAPR Asian Conference on Pattern Recognition (ACPR), pp 695-699. doi:10.1109/ACPR.2015.7486592

37. Gidaris S, Komodakis N (2015) Object detection via a multi-region and semantic segmentation-aware CNN model. In: Proceeding of 2015 IEEE International Conference on Computer Vision (ICCV), pp 1134-1142. doi:10.1109/ICCV.2015.135

38. Zhang X, Bao H, Ye J et al (2013) A data gathering scheme for WSN/WSAN based on partitioning algorithm and mobile sinks. In: IEEE International Conference on High Performance Computing and Communications and 2013 IEEE International Conference on Embedded and Ubiquitous Computing. IEEE: 1968-1973

39. Sung Y, Cho K (2012) Development and evaluation of wireless 3D video conference system using decision tree and behavior network. EURASIP J Wirel Commun Netw 1:1-14

40. Pimenta PC, De Freitas CM (2010) Security and privacy analysis in social network services. In: 2010 5th Iberian Conference on Information Systems and Technologies (CISTI), IEEE, (pp 1-6)

41. Zhang K, Liang X, Shen X, Lu R (2014) Exploiting multimedia services in mobile social networks from security and privacy perspectives. IEEE Commun Mag 52(3):58-65 